\title{
Comparative Study of Dermatoscopic and Histopathologic Results in Facial Basal Cell Carcinoma and Melanocytic Nevi
}

\author{
Mehdi Amirnia1, Mohammad-Reza Ranjkesh', Mahzad Azimpouran²*, Farid \\ Karkon-Shayan ${ }^{3}$, Hossein Alikhah ${ }^{4}$, Mohammadali Jafari-Asl ${ }^{5}$, Reza Piri ${ }^{6}$, \\ Mohammad Naghavi-Behzad ${ }^{7}$
}

\begin{abstract}
Background: Dermatoscopy can be applied to diagnose pigmented skin lesions. The aim of the present study was to compare dermatoscopic and histopathologic results in basal cell carcinoma (BCC) and melanocytic nevus of theface. Materials and Methods: In an analytical-descriptive study, 61 patients suspected of BCC or melanocytic nevi of face were randomly selected. The skin lesions of patients were evaluated with dermatoscopic method from February 2012 to February 2014 and results were compared with pathological features of samples. Results: In this study, mean age of patients was $49.5 \pm 18.9$. Some $25(41 \%)$ were men and $36(59 \%)$ were women. In 27 cases $(44.3 \%)$ there was diagnosis of melanocytic nevus, in 28 cases $(45.9 \%)$ diagnosis of $\mathrm{BCC}$, and in 3 cases $(4.9 \%)$ there was mixed diagnosis. The relationship between patients' gender and dermatoscopic diagnosis of the patients was statistically significant $(\mathrm{P}=\mathbf{0 . 0 0 1})$. For $\mathrm{BCC}$ the sensitivity and specificity of dermatoscopic method were $100 \%$ and $97 \%$ respectively and for melanocytic nevi $96.4 \%$ and $97 \%$. Conclusions: Dermatoscopic study not only can be helpful in improving clinical diagnosis while guiding missed malignant lesions to pathologic evaluations, but also could be useful in evaluating further suspicious or recurrent cases.
\end{abstract}

Keywords: Dermatoscopy - basal cell carcinoma - melanocytic nevi - histopathology

Asian Pac J Cancer Prev, 17 (1), 425-429

\section{Introduction}

The early history of dermatoscopy goes back to $17^{\text {th }}$ century A.D when Kohlhaus first observed matrix arteries through microscope. In $19^{\text {th }}$ century, Unna proposed the term diascopy after experiment and observation of lupus vulgaris lesions under spectacle lenses. Finally in mid $20^{\text {th }}$ century, Zeiss cooperating with Saphier presented the term dermatoscopy. Then Goldman made the first portable dermatoscope in the second half of 20th century and for the first time studied melanocytic nevi and melanoma. As it is clear, dermatoscopy or dermoscopy is one of the modern techniques in dermatology which is implemented with medium magnification in study and diagnosis of superficial and pigmented lesions of skin (Atherton and Moss, 1998; Khanage et al., 2012; Ghojazadeh et al., 2013; Tang et al., 2013; Mortazavi et al., 2014; Tschandl et al., 2015).

Nowadays advanced systems of dermatoscopes have been presented in the world. One of these advanced systems is Mole Max which is equipped with a camera alongside with probe which records images related to lesions in memory of dermatoscope. One of the cases which could be mentioned due to importance of the issue is skin melanoma (Rezze et al., 2006; Micali et al., 2011; Miteva and Tosti, 2012; Rosendahl et al., 2012).

Weismann et al in 1995 had introduced dermatoscopy as a research tool in diagnosis of skin pigmented tumors. It has been expressed in this study that it is possible to diagnose skin pigmented lesions under dermatoscopy without biopsy excision (Weismann et al., 1995). In case of success of dermatoscopy and defining definite criteria for any lesion it is possible to omit or postpone most of these diagnostic excisions. The most important lesions which clinically cause diagnostic problems or malignant melanoma include BCC, seborrheic keratosis, and melanocytic nevi (Hirokawa and Lee, 2011; Di Cesare et al., 2012; Fargnoli et al., 2012; Fakhrjou et al., 2013; Longo et al., 2014; Mansoori et al., 2014). Therefore in this study lesions suspected of BCC and melanocytic nevi of face were studied.

Since dermatoscopy diagnostic criteria are developing, thus it is possible to justify the importance of issue and novelty of investigation and study of this subject according to finding possible diagnostic criteria on above mentioned

${ }^{1}$ Department of Dermatology, ${ }^{2}$ Department of Pathology, ${ }^{3}$ Medical Philosophy and History Research Center, ${ }^{4}$ Department of Emergency Medicine, ${ }^{5}$ Medical Education Research Center, ${ }^{6}$ Liver and Gastrointestinal Diseases Research Center, ${ }^{7}$ Students' Research Committee, Tabriz University of Medical Sciences, Tabriz, Iran*For correspondence: Mahzad.a.ma@Gmail.com 
skin lesions.

Aim of this study was to determine relationship and compare dermatoscopic findings with pathologic results of biopsy in BCC and melanocytic nevi of face.

\section{Materials and Methods}

In a descriptive-analytical study from February 2012 to February 2014, we randomly selected using Rand list (version 1.2) software and investigated 67 patients suspected of BCC or melanocytic nevi of face who had referred to dermatology clinic of Sina Educational Centre of Tabriz (which is a referral center for dermatology in the North West of Iran) to excise or examine dermal lesion. In previous studies it has been cleared that dermatoscopy has been one of the noninvasive diagnostic techniques and no additional and non-normative intervention is being done.

In the first stage after clinical examination and filling demographic check list in terms of age, sex, place of lesion, and time duration of lesion creation, mentioned lesions underwent dermatoscopic evaluation. Alcohol was used to create liquid interface between lesion and dermatoscope and in dermatoscope observation first 3-point checklist and then dermatoscopic criteria of melanocytic nevi and BCC were used according to the essential dermatoscopy reference (Kaya et al., 2005) (Table 1).

In 3-point checklist section, existence of more than two criteria is considered as excision indication and investigating lesion, since our study was noninterventional, this section was only used comparatively. In other section dermatoscopic patterns of melanocytic nevi and diagnostic criteria for BCC were investigated and in case of existing additional criterion or diagnostic criterion for other lesions, they were recorded in section of other criteria.

This study was approved in Research Ethics Committee of Tabriz University of Medical science.

Collected information has been presented as mean \pm standard deviation (mean \pm SD) and also as frequency and percentage. Implemented software program is SPSSTM version 16. To compare quantitative variables Student T-test was used and for qualitative variables Chisquare and Fischer exact test if needed, have been used. Sensitivity and specificity in this study has been obtained using medcalc statistical software version 9.2. In all cases of study, results were statistically considered as significant with $\mathrm{P} \geq 0.05$.

\section{Results}

$25(41 \%)$ Of patients were men and $36(59 \%)$ were women. Mean age of patients was $49.5 \pm 18.9$ years $($ mean \pm SD). Age range of patients was 24-81. Mean age of men was $63.3 \pm 18.9$ (25-81) years and that of women was $39.8 \pm 14.5$ (24-81) years. In examined patients, mean time duration of lesion creation was 6 years and 10 months (82 months) with time range of 1 month to 20 years. In 10 cases $(16.4 \%)$ of patients lesion has existed since birth. Mean time duration of lesion creation in terms of gender difference was 119 months for women (about 10 years) in time range of 1 month to 20 years and that of men was 43 months (about 4 years) in range of 1 month to 18 years which shows early referring of men to physician.

In terms of anatomic site, mentioned lesion was in cheek area in 20 cases $(32.8 \%)$, in nasal area in 16 cases $(26.2 \%)$, in forehead area in 8 cases $(13.1 \%)$, in mandibular angle in 6 cases $(9.8 \%)$, in chin area in 6 cases $(9.8 \%)$, in upper lip area in 3 cases $(4.9 \%)$, and in area around the eye in 2 cases $(3.3 \%)$. Totally in terms of anatomic site of lesion the highest frequency was for cheek area with $32.8 \%$ and the lowest frequency was for area around eye with $3.3 \%$.

In terms of dermatoscopic criteria asymmetry solely existed in 14 cases (23\%) and blue-white structures solely exited in 1 case (1.6\%). Atypical network criterion didn't solely exist in any of the patients. 16 cases $(26.2 \%)$ had first 2 criteria, 4 cases $(6.6 \%)$ had the first and third criteria, and 3 cases (4.9\%) had all three criteria based on Tables presented in chapter three. 5 cases $(8.2 \%)$ of patients had none of the dermatoscopic criteria of 3-point checklist.

In terms of dermatoscopic diagnosis, 28 cases ( $45.9 \%$ ) had BCC diagnosis, 27 cases $(44.2 \%)$ had diagnosis of melanocytic nevi, and 3 cases $(4.9 \%)$ had mixed diagnosis. On BCC, 14 cases (50\%) had point-3, 11 cases (39.2\%) had point-2, 2 cases $(7.1 \%)$ had point-1, and 1 case (3.5\%) had none of the dermatoscopic criteria. In terms of melanocytic nevi, 12 cases $(44.4 \%)$ had point-1, 9 cases $(33.3 \%)$ had point-2, 3 cases $(11.1 \%)$ had point- 3 , and 4 cases $(14.8 \%)$ had none of the dermatoscopic criteria.

In terms of biopsy indication since existence of 2 criteria out of 3 criteria for dermatoscopy is considered as an indication for biopsy, in this study only 41 cases (67.2\%) of patients had biopsy indication. Rest of the

Table 1. 3-Point Checklist and Dermatoscopic Criteria of Melanocytic Nevi and BCC

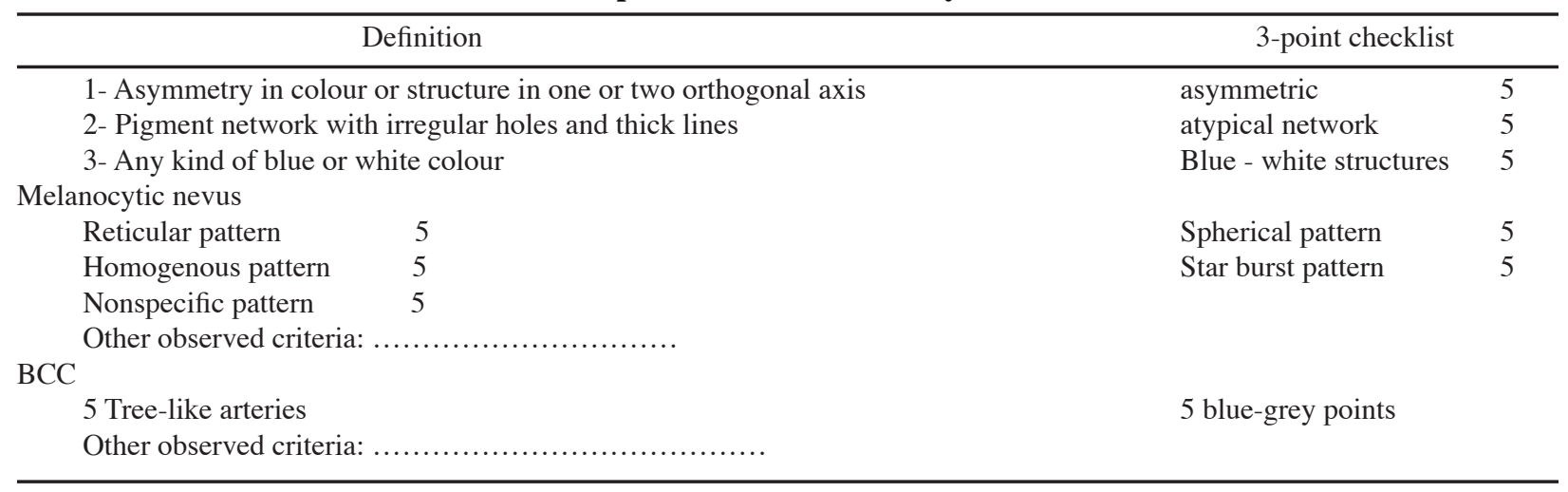


Table 2. Dermatoscopic Pattern of Melanocytic Nevi

\begin{tabular}{ll}
\hline Melanocytic nevus pattern & $\mathrm{N}=30$ \\
\hline Reticular pattern & $1(3.3 \%)$ \\
Spherical pattern & $6(20 \%)$ \\
Star burst pattern & $5(16.7 \%)$ \\
Homogenous pattern & ------ \\
Nonspecific pattern & $5(16.7 \%)$ \\
Existence of first three cases & $1(3.3 \%)$ \\
Existence of the second and fourth cases & $3(10 \%)$ \\
Existence of the first and second cases & $5(16.7 \%)$ \\
Existence of the second and third cases & $4(13.3 \%)$ \\
\hline
\end{tabular}

Table 3. Dermatoscopic Pattern of BCC

\begin{tabular}{lr}
\hline Pattern of BCC & $\mathrm{N}=31$ \\
\hline Tree-like arteries & $4(12.9 \%)$ \\
blue-grey points & $2(6.5 \%)$ \\
Existence of the first and second cases & $25(80.6 \%)$ \\
\hline
\end{tabular}

patients, i.e. 20 cases $(32.8 \%)$ had no indication for biopsy in terms of dermatoscopy. Dermatoscopic patterns of melanocytic nevi have been presented in Table 2 and dermatoscopic pattern of basal cell carcinoma has been presented in Table 3.

25 cases $(92.6 \%)$ of patients with melanocytic nevus were women and only 2 cases (7.4\%) were men. In terms of BCC 21 cases $(75 \%)$ of patients were men and only 7 cases $(25 \%)$ were women. Relationship between gender of patients and dermatoscopic diagnosis in patients was statistically significant $(\mathrm{P}=0.001)$.

Pathologic examination of biopsied samples in 28 cases $(45.9 \%)$ of patients was diagnosis of melanocytic nevi, in 27 cases $(44.3 \%)$ was BCC, in 1 case $(1.6 \%)$ was seborrheic keratosis, in 1 case $(1.6 \%)$ was reaction to foreign substance, in 1 case $(1.6 \%)$ was folliculitis associated with calcification, in 1 case was (1.6\%) abscess, and in 2 cases (3.3\%) was insitu carcinoma.

In terms of gender difference in studied women 26 cases $(72.2 \%)$ were melanocytic nevi and 9 cases $(25 \%)$ were BCC. Other previously mentioned diagnoses were also one case in women and 5 cases in men. Relationship between patient gender and pathological diagnosis after biopsy was statistically significant for patients under study $(\mathrm{P}=0.001)$.

Dermatoscopic sensitivity and specificity in this study has been obtained using medcalc statistical software version 9.2, which is as below: $i$ ). About BCC sensitivity and specificity of dermatoscopy method are $100 \%$ and $97 \%$ respectively which could be compared with pathology (biopsy) giving 100\% diagnosis. ii). About melanocytic nevi sensitivity and specificity of dermatoscopy method are $96.4 \%$ and $97 \%$ respectively which could be compared with pathology (biopsy) giving 100\% diagnosis.

\section{Discussion}

Dermatoscopy is one of the new techniques in dermatology which is used with medium magnification in investigating and diagnosing superficial and pigmented lesions of the skin. A special probe has been used in this technique where before examining the surface of skin is cleaned with oil. Using this technique it is possible to observe the range of distribution of melanin pigment in epidermis, junction between epidermis and dermis and superficial layers of dermis in higher details (Scalvenzi et al., 2008).

Now this technique is widely used in European countries like Germany, Austria, and Australia. Using dermatoscopy technique is simple but diagnosis of patterns related to diseases needs enough skill, experience, and training.

From special cases which could gain benefits of dermatoscopy is that of evaluating multiple atypical nevi and large non-excised congenital nevi and also evaluating primary small BCCs in non-vital areas and also early diagnosis of $\mathrm{BCC}$ considering its slow developmental procedure.

Nowadays in the world advanced systems have been provided for dermatoscopes. One of these advanced systems is Mole Max system which is equipped with a camera as well as a probe which records and saves images related to lesions in memory of dermatoscope.

In a comprehensive study, investigated dermatoscopic method in diagnosis of dermal pigmented tumors. In this study while it has been mentioned that it is possible to implement dermatoscopy for evaluating epidermis pigmented structures, junction of dermis-epidermis and papillary dermis layer to diagnose disease, but it has also been mentioned that dermatoscopy is unable to properly reject malignant lesions. It has been concluded in this study that histopathological study could have an undisputed diagnosis in derma diseases (Weismann et al., 1995).

Another study investigated dermatoscopy in diagnosis of superficial BCC. In this investigation dermatoscopic patterns of 42 patients suffering superficial BCC were studied whose results are as below:

Pattern of shiny white to red areas existed in all patients, erosions in $78.6 \%$, pattern of short fine telangiectasias in $66.6 \%$, leaf-like areas in $16.6 \%$, arborizing telangiectasias in $14.3 \%$, blue-gray globules in $14.3 \%$, and large bluegray ovoid nests existed in $4.7 \%$ of patients. In this study it has been concluded that it is possible to use first two patterns as main diagnostic criteria for superficial BCCs. Other criteria also would be applicable in bringing up differential diagnosis if they have diagnostic value in superficial BCCs (Scalvenzi et al., 2008).

Also in our study of 31 cases (50.8\%) with possible and definite diagnosis of BCC, 4 cases (12.9\%) had pattern of arborizing vessels, 2 cases (6.5\%) had pattern of blue-gray blotches, and 25 cases (80.6\%) had both mentioned patterns.

Also a study compared dermatoscopic findings with results of histopathology like our study. In this investigation, relationship between existence of BCC dermatoscopic patterns and pathologic findings was statistically significant. Among available patterns only whitish veil pattern was not significant. In this study dermatoscopy has also been mentioned as an important diagnostic device in diagnosing pigmented basal cell carcinoma (BCC) (Demırtaşoğlu et al., 2006). 
In a similar study, investigated relationship between dermatoscopy and pathologic findings. It is stated in this study in addition to the point that dermatologist could have a direct observation of skin lesion before evaluating pathology, even as an instruction he could ask pathologist to carefully examine the parts of skin lesion which are suspected to malignancy (Kaya et al., 2005).

No study was found with more details in anatomic site of lesion and time duration of its creation and other similar variables to those investigated in our study, to compare the results. Despite previous studies, in our study we calculated sensitivity and specificity of dermatoscopy method in diagnosing BCC and melanocytic nevi of face, whose results are as below:

About BCC sensitivity and specificity of dermatoscopy method is $100 \%$ and $97 \% \%$ respectively comparing with pathology method (biopsy) providing $100 \%$ diagnosis (Johr, 2002; Wang et al., 2004; Fracaroli et al., 2013). Also considering existence of two criteria of dermatoscopy for BCC which only constituted 25 cases $(80.6 \%)$, sensitivity and specificity of dermatoscopy is $93.3 \%$ and $89.2 \%$ respectively. About melanocytic nevi sensitivity and specificity of dermatoscopy is $96.4 \%$ and $97 \%$ respectively which is comparable to pathology method (biopsy) with 100\% diagnosis (Stolz et al., 1989; de Giorgi et al., 2006; Morales-Callaghan et al., 2008).

The most important difference of this study and similar studies is difference in percentage of dermatoscopic criteria which may imply necessity for providing more exact criteriawith computer pattern to define criteria. In case of success of dermatoscopy and defining and determining specific criteria for any lesion it is possible to omit or postpone most of diagnostic excisions. Even in some cases in pathologic there is no definite diagnostic criteria such as melanoma or BCC especially in primary stages, and dermatoscopy could be very helpful in these cases (Argenziano et al., 2007).

In short dermatoscopy is a bridge between clinical diagnosis and pathologic evaluation. The only point which is needed to be remembered is that implementing dermatoscopy in severe non-crusted and ulcer lesions especially in BCC in some cases leads to problems in observing related criteria (Argenziano et al., 2003; Celebi et al., 2009; Soyer et al., 2011).

Results of this study shows that the minimum importance which could be given to dermatoscopy is that although it is not possible to replace pathology with it in determining malignancy or benignancy of lesions, but it could be used in improving accuracy of clinical diagnosis and leading missed malignant lesions toward pathological examination and even in later investigating of suspicious or recurrent. Of course it should be mentioned that dermatoscopy is not a definite diagnostic method and conducting biopsy in all suspicious cases is necessary (Boldrick et al., 2007; Soyer et al., 2011).

Conclusion: Results of this study shows that the minimum importance which could be given to dermatoscopy is that although it is not possible to replace pathology with it in determining malignancy or benignancy of lesions, but it could be used in improving accuracy of clinical diagnosis and leading missed malignant lesions toward pathological examination and even in later investigating of suspicious or recurrent.

Proposals: $i$ ). Conducting more studies on defined criteria for melanocytic nevi and BCC. ii) Conducting dermatoscopic studies on other pre-malignant or malignant lesions and defining diagnostic criteria for them. iii). Equipping dermatoscopes with computer and photography to more accurate evaluation of mentioned criteria.

\section{References}

Argenziano G, Soyer HP, Chimenti S, et al (2003). Dermoscopy of pigmented skin lesions: results of a consensus meeting via the Internet. J Am Acad Dermatol, 48, 679-93.

Argenziano G, Zalaudek I, Ferrara G, et al (2007). Dermoscopy features of melanoma incognito: indications for biopsy. $J \mathrm{Am}$ Acad Dermatol, 56, 508-13.

Atherton D, Moss C (1998). Naevi and other developmental defects. Rook's Textbook of Dermatology, Seventh Edition, 569-682.

Boldrick JC, Layton CJ, Nguyen J, et al (2007). Evaluation of digital dermoscopy in a pigmented lesion clinic: clinician versus computer assessment of malignancy risk. J Am Acad Dermatol, 56, 417-21.

Celebi ME, Iyatomi H, Schaefer G, et al (2009). Lesion border detection in dermoscopy images. Computerized Med Imag Graphics, 33, 148-53.

de Giorgi V, Sestini S, Massi D, et al (2006). Dermoscopy for "true" amelanotic melanoma: a clinical dermoscopicpathologic case study. J Am Acad Dermatol, 54, 341-4.

Demırtaşoğlu M, Ilknur T, Lebe B, et al (2006). Evaluation of dermoscopic and histopathologic features and their correlations in pigmented basal cell carcinomas. J Eur Acad Dermatol Venereol, 20, 916-20.

Di Cesare A, Sera F, Gulia A, et al (2012). The spectrum of dermatoscopic patterns in blue nevi. J Am Acad Dermatol, 67, 199-205.

Fakhrjou A, Dastranj-Tabrizi A, Ghojazadeh M, et al (2013). Diagnostic Value of Protein Ki67 (MIB-1) in Atypical Pap Smears of Postmenopausal Women. Asian Pac J Cancer Prev, 14, 4815-8.

Fargnoli MC, Kostaki D, Piccioni A, et al (2012). Dermoscopy in the diagnosis and management of non-melanoma skin cancers. Eur J Dermatol, 22, 456-63.

Fracaroli TS, Lavorato FG, Maceira JP, et al (2013). Parallel ridge pattern on dermoscopy: observation in non-melanoma cases. Anais Brasileiros De Dermatologia, 88, 646-8.

Foroughi MR, Razi A, Golforoushan F (2008). Study on the results of surgical treatment of basal cell carcinoma on the scalp by secondary intention healing. Med J Tabriz Univ Med Sci, 30, 97-104.

Ghojazadeh M, Mohammadi M,Azami-Aghdash S, et al (2013). Estimation of cancer cases using capture-recapture method in Northwest Iran. Asian Pac J Cancer Prev, 14, 3237-41.

Hirokawa D, Lee JB (2011). Dermatoscopy: an overview of subsurface morphology. Clinics Dermatology, 29, 557-65.

Johr RH (2002). Dermoscopy: alternative melanocytic algorithms-the $\mathrm{ABCD}$ rule of dermatoscopy, menzies scoring method, and 7-point checklist. Clinics dermatol, 20, 240-7.

Khanage SG, Raju SA, Mohite PB, et al (2012). Synthesis and pharmacological evaluation of some new pyrimidine derivatives containing 1, 2, 4-triazole. Advanced Pharm Bull, 2, 213 .

Kaya G, Braun R, Marghoob A, et al (2005). Histopathological correlation in dermoscopy. Atlas of Dermoscopy, 23-41.

Longo C, Zalaudek I, Moscarella E, et al (2014). Clonal 
seborrheic keratosis: dermoscopic and confocal microscopy characterization. J Eur AcadDermatol Venereol, 28, 1397400.

Lotfinegad P(2014). Immunomodulatory nature and site specific affinity of mesenchymal stem cells: a hope in cell therapy. Advanced Pharm Bull, 4, 5.

Mansoori B, Shotorbani SS, Baradaran B (2014). RNA Interference and its Role in Cancer Therapy. Advanced Pharm Bull, 4, 313.

Micali G, Lacarrubba F, Massimino D, et al (2011). Dermatoscopy: alternative uses in daily clinical practice. $J$ Am Acad Dermatol, 64, 1135-46.

Miteva M, Tosti A (2012). Hair and scalp dermatoscopy. J Am Acad Dermatol, 67, 1040-8.

Morales-Callaghan A, Castrodeza-Sanz J, Martinez-Garcia G, et al (2008). Correlation between clinical, dermatoscopic, and histopathologic variables in atypical melanocytic nevi. Actas Dermo-Sifiliograficas, 99, 380-9.

Mortazavi H, Baharvand M, Mehdipour M (2014). Oral potentially malignant disorders: an overview of more than 20 entities. J Res, Dental Clinics, Dental Prospects, , 8, 6.

Rezze GG, Sa BCSd, Neves RI (2006). Dermoscopy: the pattern analysis. Anais Brasileiros de Dermatologia, 81, 261-8.

Rosendahl C, Cameron A, McColl I, et al (2012). Dermatoscopy in routine practice:'Chaos and clues'. Australian Family Physician, 41, 482.

Scalvenzi M, Lembo S, Francia MG, et al (2008). Dermoscopic patterns of superficial basal cell carcinoma. International $J$ Dermatol, 47, 1015-8.

Soyer HP, Argenziano G, Hofmann-Wellenhof R, et al (2011). Dermoscopy: the essentials, Elsevier Health Sciences.

Stolz W, Bilek P, Landthaler M, et al (1989). Skin surface microscopy. Lancet, 334, 864-5.

Tang L, Zhang W, Su B, et al (2013). Long noncoding RNA HOTAIR is associated with motility, invasion, and metastatic potential of metastatic melanoma. Bio Med Res Int, 2013.

Tschandl P, Rosendahl C, Kittler H (2015). Dermatoscopy of flat pigmented facial lesions. Je Eur Academy Dermatol Venereol, 29, 120-7.

Wang SQ, Kopf AW, Koenig K, et al (2004). Detection of melanomas in patients followed up with total cutaneous examinations, total cutaneous photography, and dermoscopy. $J$ Am Acad Dermatol, 50, 15-20.

Weismann K, Osterlind A, Thomsen H (1995). Dermatoscopy. An investigative method for the diagnosis of pigmented skin tumors. Ugeskrift for laeger, 157, 147-52. 